\title{
EFICIENCIA DEL FORWARD COMO INSTRUMENTO DE COBERTURA DEL RIESGO CAMBIARIO EN LAS EMPRESAS QUE REALIZAN OPERACIONES DE COMERCIO EXTERIOR, 2011-2017*
}

Recibido: 24 de abril de 2019 • Aprobado: 15 de mayo de 2019

https://doi.org/10.22395/seec.v22n51a3

Carlos Andrés Díaz Restrepo**

Marlen Isabel Redondo Ramírez ${ }^{* * *}$

\section{RESUMEN}

Este artículo evalúa la eficiencia de los contratos forward derivados de la tasa de cambio dólares americanos/pesos colombianos (USD/COP), como instrumentos de cobertura de riesgo cambiario, al cual están expuestas las empresas que realizan operaciones en divisas. Para ello se analizaron los precios spot y forward USD/COP entre los años 2011 y 2017, disponibles en la Bolsa de Valores de Colombia, valorando su riesgo a través del VaR (value at risk) y evaluando sus impactos como instrumento de cobertura en riesgo de tasa de cambio.

Además de la validación empírica del forward como instrumento de cobertura, se encontraron algunas ineficiencias de este instrumento financiero, debido a su baja disponibilidad y a los altos costos de transacción en el uso de este derivado como instrumento de cobertura.

\section{PALABRAS CLAVE}

Comercio internacional; forwards; riesgo financiero; riesgo de tasa de cambio; riesgo cambiario.

\section{CLASIFICACIÓN JEL}

G11, G14, G23, G32

\section{CONTENIDO}

Introducción; 1. Tipo de cambio; 1.1. Tipos de cambio y mercado de divisas; 2. Riesgo cambiario; 2.1 . Sistema financiero colombiano; 3. Metodología; 3.1. Recopilación de la información; 4. Resultados; 4.1. Análisis del valor spot peso/dólar entre el 2011 y 2017; 3.2. Análisis del precio forward peso/ dólar entre el 2011 y 2017; 5. Conclusiones; Bibliografía.

\footnotetext{
Artículo resultado de los hallazgos encontrados en el proyecto de investigación Gestión del riesgo en tasa de cambio de las empresas del cluster textil juntos, adscrito al Grupo de Investigación en Estudios Económicos y de la Administración (clasificado en categoría C en Colciencias 2017) y el Grupo de Investigación Tendencia Económica Mundial (clasificado en categoría C en Colciencias 2017), financiado por la Universidad Libre, seccional Pereira y la Universidad Católica de Pereira, desarrollado en los años 2017 y 2018.

.* Administrador de empresas, Universidad Cooperativa de Colombia, Pereira, Colombia. Magíster en Administración de Negocios, Universidad Autónoma de Manizales, Manizales, Colombia. Estudiante de Doctorado en Administración, Universidad Nacional, Manizales, Colombia. Profesor de la Facultad de Ciencias Económicas y Administrativas, Universidad Católica de Pereira, Pereira, Colombia. Correo electrónico: carlos. diaz@ucp.edu.co

"* Economista, Universidad Católica de Pereira, Pereira, Colombia. Magíster en Administración de Negocios, Universidad Autónoma de Manizales, Manizales, Colombia. Estudiante del Doctorado en Ciencias de la Educación, Universidad de Cuauhtémoc, México. Profesora de la Facultad de Ciencias Económicas, Administrativas y Contables, Universidad Libre, Pereira, Colombia. Correo electrónico: isabel.redondo@ unilibre.edu.co
} 


\title{
EFFICIENCY OF FORWARD AS AN INSTRUMENT FOR EXCHANGE RISK COVERAGE IN COMPANIES PERFORMING FOREIGN TRADE OPERATIONS, 2011-2017
}

\begin{abstract}
This paper evaluates the efficiency of forward contracts derived from the exchange rate of American dollars / Colombian pesos (USD / COP), as a hedging instrument for foreign exchange risk, to which companies that carry out foreign exchange operations are exposed. For this, the USD / COP spot and forward prices between 2011 and 2017, available in the Colombian Stock Exchange, were analyzed, valuing their risk through the VaR (value at risk) and their impacts as a hedging instrument of risk of exchange rate.

In addition to the empirical validation of the forward as a hedging instrument, some inefficiencies of this financial instrument were found, due to its low availability and the high transaction costs in the use of this derivative as a hedging instrument.
\end{abstract}

\section{KEYWORDS}

International trade; forwards; financial risk; exchange rate risk; foreign exchange risk.

JEL CLASSIFICATION

G11, G14, G23, G32

\section{CONTENTS}

Introduction; 1. Exchange rate; 1.1. Exchange rates and foreign exchange market; 2 . Foreign exchange risk; 2.1. Colombian financial system; 3. Methodology; 3.1. Information recollection; 4. Results; 4.1 . Analysis of the peso / dollar spot value between 2011 and 2017; 4.2. Analysis of the forward peso / dollar price between 2011 and 2017; 5. Conclusions; Bibliography.

\section{EFICIÊNCIA DO FORWARD COMO INSTRUMENTO DE COBERTURA DO RISCO CAMBIAL NAS EMPRESAS QUE REALIZAM OPERAÇÕES DE COMÉRCIO EXTERIOR, 2011-2017}

\section{RESUMO}

Este artigo avalia a eficiência dos contratos forward derivados da taxa de câmbio dólares americanos/ pesos colombianos (USD/COP), como instrumentos de cobertura de risco cambial, ao qual estão expostas as empresas que realizam operações em divisas. Para isso, foram analisados os preços spot e forward USD/COP entre 2011 e 2017, disponíveis na Bolsa de Valores da Colômbia, avaliando seu risco por meio do VaR (value at risk) e avaliando seus impactos como instrumento de cobertura em risco de taxa de câmbio. Além da validação empírica do forward como instrumento de cobertura, foram verificadas algumas ineficiências desse instrumento financeiro, devido à sua baixa disponibilidade e aos altos custos de transação no uso desse derivado como instrumento de cobertura.

\section{PALAVRAS-CHAVE}

Comércio internacional; forwards; risco financeiro; risco de taxa de câmbio; risco cambial.

\section{CLASSIFICAÇÃO JEL}

\author{
G11, G14, G23, G32
}

\section{CONTEÚDO}

Introdução; 1. Tipo de câmbio; 1.1. Tipos de câmbio e mercado de divisas; 2. Risco cambial; 2.1. Sistema financeiro colombiano; 3. Metodologia; 3.1. Recopilação da informação; 4. Resultados; 4.1. Análise do valor spot peso/dólar entre 2011 e 2017; 3.2. Análise do preço forward peso/dólar entre 2011 e 2017; 5. Conclusões; Bibliografia. 
Eficiencia del forward como instrumento de cobertura del riesgo cambiario en las empresas que realizan operaciones...

\section{INTRODUCCIÓN}

Desde el año 1999, Colombia adoptó un régimen cambiario en el que la tasa de cambio USD/COP es de libre fluctuación, esto significa que los precios de cotización en el mercado de esta divisa dependen de la oferta y la demanda existente, contando en algunas ocasiones con intervención del Banco de la República a través de compra o venta de divisas (dólar), con el fin de regular un poco los precios.

Estas fluctuaciones en la tasa de cambio afectan los activos y los pasivos de las empresas que realizan algún proceso de internacionalización, debido a la constante exposición a procesos de inversión (cartera) o de financiación (proveedores). De acuerdo a Kozikowski (2013), el riesgo cambiario se refiere a la incertidumbre generada por el precio de cotización de una divisa en el futuro, pudiendo afectar los precios de los activos y los pasivos en moneda extranjera, al momento de aplicarlos a moneda local. En la misma línea Jacque (2014), define el concepto de exposición a tasa de cambio como los montos en moneda extranjera de una empresa que está constantemente envuelta en procesos de importaciones y exportaciones, dados por las fluctuaciones de los precios de las divisas en la que se realiza la negociación. Finalmente Grath (2016), referencia el riesgo cambiario como la afectación en precios de la moneda local por la aplicación de la tasa de cambio en operaciones de compra y venta en moneda extranjera.

De ahí que las empresas que realizan operaciones en divisas, para nuestro caso dólar, deban incluir operaciones para gerenciar el riesgo financiero de tasa de cambio USD/COP a través de instrumentos financieros, tales como los contratos de forwards de tasa de cambio, con el fin de mitigar los costos de transacción. Debido a que son pocas las empresas que conocen estas herramientas y carecen de un departamento financiero (hipótesis apriorística), que implemente estrategias conducentes a la mitigación del riesgo en tasa de cambio USD/COP.

Este artículo presenta un análisis del derivado forward de tasa de cambio USD/ COP, con el fin de usarlo como instrumento de cobertura y equilibrar el riesgo cambiario. Se debe agregar que, en el caso de las empresas que realizan operaciones de comercio exterior, tienen un alto grado de vulnerabilidad al enfrentarse al mercado internacional, es por eso por lo que se consideraron como objeto de estudio, con el fin de coadyuvar a su permanencia en el tiempo.

Para el análisis, se tomaron precios de cierre del mercado spot y forward USD/ COP, con la información disponible a través de la plataforma de la Bolsa de Valores de Colombia durante los años 2011 a 2017 usando el modelo de valor en riesgo y las medidas de varianzas y covarianzas, se verificó el comportamiento de ambos 
activos en las series de tiempo 0 a 1 mes, de 1 a 3 meses, de 3 a 6 meses y mayor a seis meses.

Entre los principales resultados de la investigación se encuentra el incremento de la exposición al riesgo en tasa de cambio relacionado con el tiempo, es decir, entre más tiempo exista entre la fecha del contrato y la fecha del pago de la divisa, mayor es la exposición al riesgo dado por las variaciones de la tasa de cambio. Además de esto, la baja disponibilidad del activo forward en la Bolsa de Valores de Colombia, condiciona su uso como instrumento de cobertura.

Finalmente, el comportamiento del valor spot de la divisa USD/COP es diferente al comportamiento de los contratos forward derivados de la tasa de cambio USD/ $\mathrm{COP}$, generando un aumento en los costos de transacción en las operaciones de importaciones o exportaciones en dólares.

\section{TIPO DE CAMBIO}

De acuerdo a Krugman y Obstfeld (2012) y a Kozikowski (2013), el tipo de cambio está definido por el precio de una moneda en función de otra, en nuestro caso podríamos nombrarlo como la cantidad de pesos colombianos (COP) que debemos pagar por cada dólar estadounidense (USD). Debido a que el tipo de cambio tiene una variación de acuerdo a la oferta y a la demanda de la moneda extranjera (Banco de la República, 2013), también condiciona los precios de los activos financieros y tiene un papel fundamental en el comercio internacional dado que permite comparar los productos y servicios producidos en diferentes países.

De acuerdo a Krugman (2012), las variaciones del tipo de cambio también se definen como apreciaciones y depreciaciones. Una depreciación del peso colombiano frente al dólar estadounidense se da cuando debemos pagar más pesos por dólar, al contrario de esto, cuando debemos pagar menos pesos colombianos por cada dólar estadounidense nos enfrentamos a una apreciación del peso frente al dólar.

De acuerdo con lo anterior, podemos deducir que los tipos de cambio permiten fijar los precios internacionales de bienes y servicios y comparar los precios nacionales con los extranjeros.

\subsection{Tipos de cambio y mercado de divisas}

La compra y venta de divisas es la funcionalidad que generan las finanzas internacionales para que, a través del tipo de cambio, se puedan comprar y vender divisas en cualquier parte del mundo, el precio lo determina el mercado de divisas, es decir, 
Eficiencia del forward como instrumento de cobertura del riesgo cambiario en las empresas que realizan operaciones...

este mercado condiciona los demás mercados financieros internacionales, debido a que le asigna los precios de cambio de las divisas.

En Colombia la Dirección de Impuestos y Aduanas Nacionales (DIAN, 2019), menciona el mercado de divisas conformado por la Bolsa de Valores de Colombia, por los intermediarios del mercado cambiario y por las personas y empresas que se ven en la necesidad de transferir el poder adquisitivo de una divisa al peso colombianos a través del tipo de cambio.

Además de tener la característica de cualquier mercado en el que constantemente existen compradores y vendedores, este representa el $75 \%$ de las operaciones interbancarias internacionales, y el 89 \% de las transacciones son realizadas en dólares, como lo afirman García y Díez (2014).

El mercado de divisas condiciona los precios de los tipos de cambio de las divisas, debido a que enfrenta las ofertas entre los compradores y los vendedores que ofertan su divisa y compran otra, como lo expone Kozikowski (2013).

\section{RIESGO FINANCIERO Y RIESGO CAMBIARIO}

El riesgo financiero lo podemos definir como los cambios en las tasas de interés o los costos de capital en una moneda local. Este tipo de riesgo expone a que una empresa pueda caer en impagos dada por la probabilidad de que un negocio no genere los excedentes necesarios o de que los resultados financieros no sean los planeados inicialmente, como lo describe Grath (2016).

Todas las empresas están expuestas a riesgos, más aún cuando están desarrollando procesos de internacionalización. Su perfil de riesgo puede variar por el incremento en el desarrollo de mercados domésticos o internacionales, esta es la principal razón por la que las empresas deben identificar sus riesgos, entre estos el de tasa de cambio, con el fin de gestionar procesos para minimizar su impacto en las finanzas de la empresa.

De acuerdo a Ye et al. (2014) el riesgo financiero se refiere a la incertidumbre sobre el valor económico de la compañía en el futuro. Este valor se puede ver afectado por cambios en las tasas de interés, procesos de devaluación, tasas de inflación o por las variaciones en la tasa de cambio de las divisas locales, comparados con las foráneas.

Dentro de los riesgos a los que más está expuesta una empresa que desarrolle procesos de internacionalización, se encuentra el riesgo en tasa de cambio, dado por un riesgo de cambio positivo o negativo en el valor económico de la empresa 
en el futuro, determinado por una variación en la tasa de cambio de la moneda local con una divisa en la que se tengan inversión o financiación. Grath (2016) la describe como la disminución del ingreso en ventas del exportador o el aumento de los costos del importador, generado por una variación en la tasa de cambio entre el día de la transacción (compra o venta) y el día del pago. Finalmente, Grath (2016) define la exposición del riesgo en tasa de cambio, como el valor del monto de la transacción en términos de la divisa extranjera, lo que condiciona el tamaño del riesgo al que se expone la empresa.

Basados en lo anterior, podemos resumir que los riesgos financieros están relacionados con la probabilidad y el impacto que tienen las variaciones no previstas en las variables macroeconómicas sobre el flujo de caja de la empresa y, por ende, en el valor de esta.

\subsection{Sistema financiero colombiano}

Según Uribe (2006, p. 1) el sistema financiero colombiano está conformado por el mercado monetario, de divisas, de capitales y otros mercados. Las principales funciones de este mercado son la facilitación de la financiación de los emisores de deuda en el corto plazo, además de brindar apoyo a la ejecución eficaz de la política monetaria y la formación temporal de tasas de interés.

El mercado de divisas, descrito por Carbaugh (2016) hace referencia a aquel escenario de mayor liquidez en el mundo, en este, los individuos, empresas, gobiernos y bancos compran y venden divisas y otros instrumentos de deuda; este mercado funciona en tres niveles, los cuales son 1) las transacciones entre los clientes exportadores, importadores, inversionistas o turistas y bancos comerciales; 2) los corredores de divisas o brokers; por medio del mercado interbancario nacional y finalmente, 3) el intercambio de divisas entre bancos comerciales y aquellas sucursales o corresponsales en el extranjero.

Los dos grandes componentes del mercado de capitales son el bancario' (intermediado) y el de valores (desintermediado), descrito por la Superintendencia Financiera de Colombia (2017). El mercado intermediado colombiano, se conforma de establecimientos de crédito, sociedades de servicios financieros, inversionistas

\footnotetext{
1 Al ser el dinero, el elemento clave para el funcionamiento del sistema financiero bancario, es de aclarar que en la actualidad, "la cantidad de dinero creada en la economía depende en últimas de la política monetaria del banco central. En tiempos normales, esto se lleva a cabo fijando tasas de interés (...) En la economía moderna, la mayor parte del dinero toma la forma de depósitos bancarios (...) la principal manera de crearlos son los préstamos que hacen los bancos comerciales. Cada vez que un banco hace un préstamo, crea simultáneamente un depósito correspondiente en la cuenta bancaria del prestatario, con lo cual crea dinero nuevo" (McLeay, Radia y Thomas, 2015, p. 355).
} 
Eficiencia del forward como instrumento de cobertura del riesgo cambiario en las empresas que realizan operaciones...

institucionales y demás organizaciones evidenciadas en la gráfica 1. Los primeros tienen como función principal canalizar y captar los recursos superavitarios de un sector, para transferirlos a aquellos con déficit por medio de colocaciones de préstamos, anticipos u operaciones.

Sobre esto, Uribe (2013) señala que las sociedades de servicios financieros son intermediadoras con los recursos del público, no ejecutan la captación a través de depósitos y la colocación no es a través de créditos; puesto que su función principal es prestar asesoría financiera especializada, para la administración de recursos. Por otra parte, aquellas instituciones que tienen como función recolectar los recursos disponibles de los agentes e invertirlos en títulos valor; se conocen como los inversionistas institucionales. Por último, Fradique-Méndez (2014) nombra a aquellas entidades que no están contempladas dentro de los grupos anteriores; pero que prestan servicios complementarios y conexos a la actividad financiera, se clasifican como otras instituciones.

\section{Gráfica 1. Sistema bancario en Colombia}
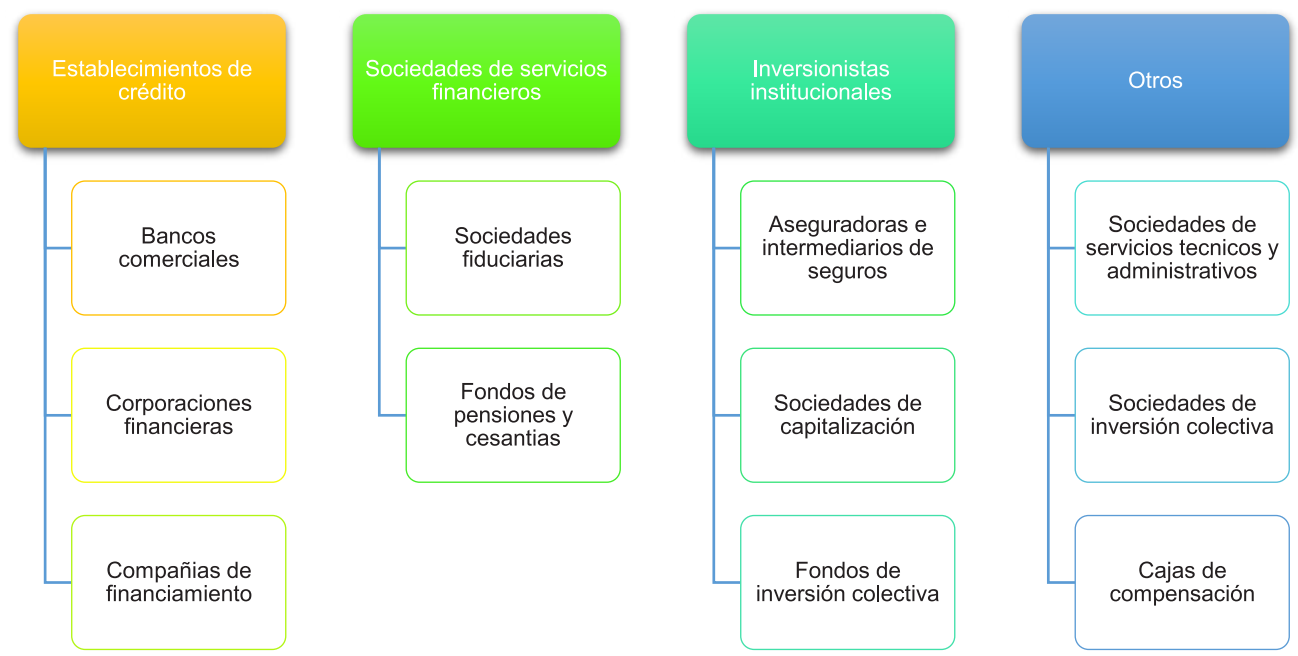

Fuente: elaboración propia a partir de Banrep (2013)

La Bolsa de Valores de Colombia (2008, p. 24), define el mercado de valores como "el conjunto de agentes, instituciones, instrumentos y formas de negociación que interactúan facilitando la transferencia de capitales para la inversión a través de la negociación de valores"; se compone de los emisores de valores, los inversionistas, los intermediadores y los diferentes proveedores de infraestructura. Los emisores de mercado son aquellas entidades que ponen en circulación títulos valores de cualquier ámbito y se encuentran inscritos en el Registro Nacional de Valores y Emisores; por su 
parte, los inversionistas, son todas aquellas personas naturales o jurídicas que invierten sus recursos en títulos valores. Un intermediario de valores es aquel ente encargado del acercamiento entre los demandantes y oferentes para la negociación de títulos valores. Por último, los proveedores de infraestructura para el mercado colombiano son el Deposito Centralizado de Valores de Colombia (Deceval), la $\mathrm{BVC}^{2}$, las sociedades calificadoras de riesgo y el Autorregulador del Mercado de Valores (AMV).

La segunda clasificación (Fradique-Méndez, 2014) de los títulos compete a la manera de establecer los rendimientos. Estos se pueden determinar desde el momento de la negociación en renta fija o al momento de la liquidación del valor futuro en renta variable. Finalmente, de acuerdo con la forma en que se trasfieran los valores, estos pueden ser nominativos que implican la inscripción del tenedor en el registro que maneje el emisor, a la orden solo del que es tenedor legítimo, es decir, la persona que ha recibido el valor y que aparece como endosatario; y los valores al portador que requieren exclusivamente la entrega, para ser legítimamente transferidos a la persona que lo reciba.

La tercera categoría (Agudelo, 2014) son los valores comúnmente transados, entre ellos se encuentran los derechos de participación en fondos de inversión colectiva, los certificados de depósito de mercancías y los contratos sobre derivados; los fondos bursátiles de inversión (ETF), estos últimos destacan por la diversificación, disminución en el tiempo de selección, seguimiento y balanceo del portafolio debido a que ofrecen carteras colectivas con "menores costos de administración, liquidez inmediata y mayor transparencia en cuanto al índice, precio o divisa de referencia"; la réplica del índice subyacente puede ser de manera directa o física, es decir, la compra directa total o parcial de los valores del índice, A su vez, puede recurrir al uso de instrumentos financieros derivados de manera indirecta o sintética, como lo señala la Comisión Nacional del Mercado de Valores (2015).

Finalmente, aquellas acciones no comprendidas en el mercado de capitales, monetario o de divisas, se catalogan como otros mercados financieros.

\footnotetext{
2 El 15 de febrero de 2017 la BVC y el Deceval firmaron un memorando de entendimiento para una posible integración corporativa, el 22 de marzo de 2017 se emitió la guía explicativa de la integración corporativa Deceval-BVC con el fin de "generar eficiencias en la cadena de valor del mercado de capitales colombiano para reducir costos y mejorar la oferta de servicios". Finalmente, el 15 de diciembre de 2017 se anunció que la BVC y Deceval serán una sola organización; después del cierre de la transacción y el intercambio de acciones entre las dos entidades, sin embargo a la fecha (28 de febrero de 2018) no se posee ningún manual que cambie las funciones de ambas entidades, por lo cual se evidencian en el texto por separado (BVCb, 2017, p. 3).
} 
Eficiencia del forward como instrumento de cobertura del riesgo cambiario en las empresas que realizan operaciones...

\section{METODOLOGÍA}

La recolección de datos fue realizada a través de la plataforma Set fx, encargada de registrar todas las operaciones del mercado de divisas en Colombia. Fueron tomados los precios spot de cotización al cierre de la tasa de cambio USD/COP entre los años 2011 al 2017. Así mismo, se tomaron los datos de cotización de cierre de los forwards derivados de tasa de cambio USD/COP, de 0 a 1 mes, de 1 a 3 meses, de 3 a 6 meses y mayores a 6 meses, para el mismo periodo.

En total se realizaron 4.573 observaciones en las que se compararon los precios de cierre de los diferentes contratos de forward, frente a los precios de cierre del valor spot de tasa de cambio USD/COP, con la finalidad de valorar su eficiencia como instrumento de cobertura a través del modelo probabilístico VaR (Value at Risk)

El uso del modelo VaR, ha sido usado para medir la posibilidad de pérdida de un portafolio de inversión con un determinado nivel de incertidumbre, en nuestro caso del $5 \%$ (Jorion, 2000), es decir con los datos calculados con precios diarios en esta investigación, una vez cada 20 días los precios estarán por debajo del valor calculado, lo que facilita la gestión del riesgo en tasa de cambio .

Analíticamente, el VaR define el límite superior de la integral en función de los retornos esperados y los podemos describir en la siguiente fórmula:

$$
\mathrm{VaR}=\propto * \sqrt{\delta^{2} * \Delta t}
$$

En la ecuación (1) $\propto$ determina la posible área de exposición de riesgo, $\delta^{2}$ la varianza de los precios de los activos y $\Delta t$ los tiempos de exposición del riesgo, de aquí se toma el límite superior de las exposiciones a los riesgos en tasa de cambio, tal como lo presenta Jorion (1990).

El estudio consistió en una validación empírica con el fin de caracterizar la realidad de las empresas expuestas a operaciones en divisas, donde se analizaron las series históricas de la tasa de cambio peso/dólar, para luego proceder a realizar los cálculos y análisis del impacto en las operaciones de comercio exterior.

Algunos de los riesgos que pueden condicionar la preservación de las empresas, se puede mencionar el riesgo de negocio, que es afectado por las decisiones que se toman en el normal funcionamiento de la empresa, como el tamaño de la producción, la asignación de precios, canales de distribución, entre otros. Al tiempo podemos mencionar el riesgo financiero, condicionado por los precios de los activos y la capacidad de la empresa de cubrir sus costos. Como ejemplo podemos mencionar el estudio realizado por Toro et al. (2015) en el que identificaron que el 73,44 \% de las empresas de la ciudad de Medellín presentaron riesgo financiero en el año 2013. 
La aplicación del modelo VaR para estimar el riesgo en tasa de cambio ha sido utilizado a través de los años en varias investigaciones a nivel mundial; Wang et al. (2010) usaron el miso modelo para medir el riesgo en tasa de cambio del Yuan chino, frente al Euro (EUR/CNY) y el Yen japonés (JPY/CNY), en este trabajo presentan la exposición al riesgo en tasa de cambio por una continua apreciación del Yuan. Zhou Li et al. (2013), lo aplicaron para valorar el riesgo de cambio de los precios en los activos de las entidades financieras, frente a la tasa de cotización del renmimbi (RNB), en su artículo evalúan la capacidad de pago de las entidades bancarias frente a las posibles pérdidas por las variaciones en la tasa de cambio. Finalmente Raúl de Jesús et al. (2013), usaron el VaR para medir el riesgo de las pérdidas potenciales de las inversiones frente a los cambios en la tasa de cambio del peso mexicano frente al dólar (USD/MXN).

\subsection{Recopilación de la información}

Una vez obtenida la información de los precios spot y forward de la tasa de cambio USD/COP, se realizaron las mediciones de los activos de 0 a 1 mes, de 1 a 3 meses, de 3 a 6 meses y mayor a 6 meses, con la información contenida en la herramienta Set Fx de la Bolsa de Valores de Colombia. Se extrajeron las cifras evidenciadas para los años 2011 a 2017 y se calcularon diferentes indicadores que midieron la exposición al riesgo, a su vez, se tomaron textos indexados y libros con el fin de construir las bases teóricas de la investigación y las plataformas mencionadas con anterioridad.

Las fases de la investigación fueron: i) preparación de información: tuvo como objetivo la preparación por medio de la exploración y la revisión de antecedentes internacionales, nacionales y regionales, además referentes teóricos que permitieron construir las bases para llevar a cabo el planteamiento del problema y el primer paso para el desarrollo de la investigación, ii) se llevó a cabo la elaboración y aplicación de instrumentos para sistematizar el comportamiento del valor spot USD/COP y los forward durante los años 2011-2017; iii) finalmente se analizó la información obtenida para determinar la eficiencia de los instrumentos financieros forward en USD/COP como instrumento de cobertura frente al riesgo de tasa de cambio.

\section{RESULTADOS}

A continuación, presentaremos el comportamiento del precio spot de cierre del USD/COP y los precios de cotización de forward USD/COP de 0 a 1 mes, de 1 a 3 meses, forward de 3 a 6 meses y activos de forward USD/COP mayores a 6 meses, basados en los datos reportados por la plataforma Set Fx de la Bolsa de Valores de Colombia. 
Eficiencia del forward como instrumento de cobertura del riesgo cambiario en las empresas que realizan operaciones...

\subsection{Análisis del valor spot peso/dólar entre el 2011 y 2017}

El análisis a través del modelo de varianzas (VaR) mostró el comportamiento tomando los precios spot de cierre reportados a través de la plataforma Set Fx, con un total de 1.808 días observados con operaciones en tasa de cambio USD/COP, durante los años 2011 a 2017.

En la tabla 1, describimos la exposición de riesgo que tuvieron las empresas en términos vencimiento diario, mensual, trimestral y semestral, con el fin de compararlos con los instrumentos de cobertura forward disponibles en la Bolsa de Valores de Colombia.

Tabla 1. Resumen de exposición al riesgo en tasa de cambio

\begin{tabular}{lccc}
\hline Vencimiento & Exposición a riesgo & Vencimiento & Exposición a riesgo \\
\hline Diario & $+1,93 \%$ & Trimestral & $\pm 13,31 \%$ \\
\hline Mensual & $\pm 8,94 \%$ & Semestral & $\pm 17,17 \%$ \\
\hline
\end{tabular}

Fuente: elaboración propia a partir de Set Fx.

De acuerdo con el resumen presentado en la tabla 1, a medida que se extiende el vencimiento, es mayor la exposición al riesgo. En caso de que una empresa presente un vencimiento de contratos a un día, enfrentó un aumento o una disminución del $1,93 \%$ en el precio de la tasa de cambio, llegando a un riesgo de un mayor o menor valor en un $17,17 \%$ para el vencimiento semestral, lo que puede afectar el flujo de efectivo de las empresas por un mayor valor en el precio de sus importaciones o un menor ingreso en sus ventas.

Gráfica 2. Precios spot de cierre diario, mensual, trimestral y semestral 2001 - 2017

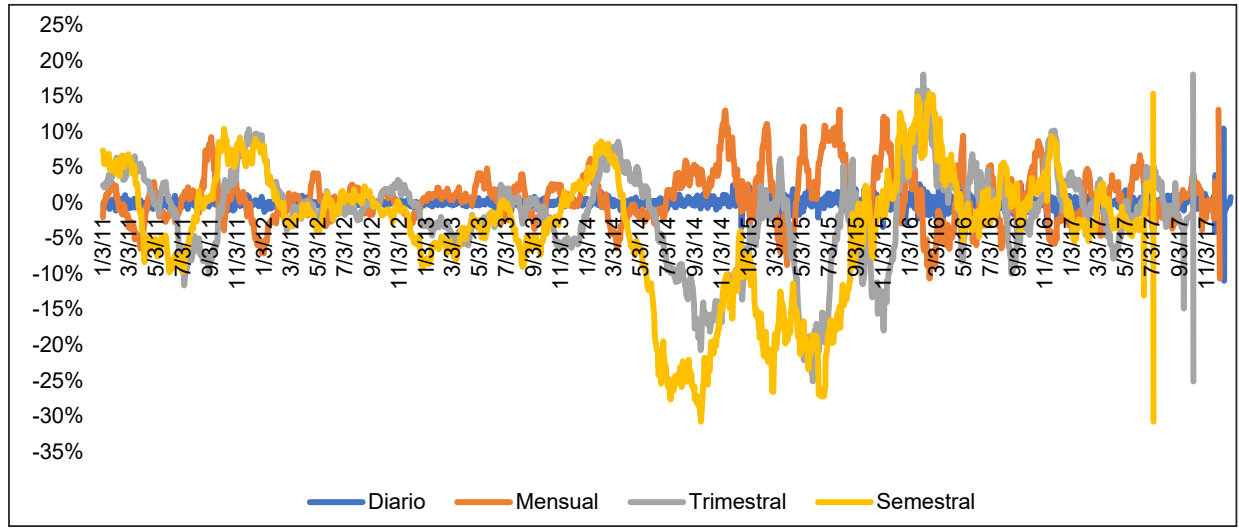

Fuente: elaboración propia a partir de Set Fx. 
En la gráfica 2, observamos el comportamiento de los precios de cierre del mercado spot USD/COP, con vencimiento diario, mensual, trimestral y semestral, podemos evidenciar que el comportamiento de los precios de cierre no es constante, además de tener una variación diferente.

Tabla 2. Resumen de variaciones máximas y mínimas diaria, mensual, trimestral y semestral 2011-2017

\begin{tabular}{|c|c|c|}
\hline Vencimiento & Variación máxima & Variación mínima \\
\hline Diario & $10,45 \%$ & $-10,95 \%$ \\
\hline Mensual & $13,14 \%$ & $-10,65 \%$ \\
\hline Trimestral & $18,09 \%$ & $-25,13 \%$ \\
\hline Semestral & $15,37 \%$ & $-30,80 \%$ \\
\hline
\end{tabular}

\section{Fuente: elaboración propia a partir de Set Fx.}

En la tabla 2, presentamos el resumen de las mayores y menores variaciones en los precios spot de cierre diario, mensual, trimestral y semestral. Durante los años 2011 a 2017, en el precio de cierre diario, la mayor variación fue del 10,45 \% y una menor variación del $-10,95$ \%; lo menos predecible de esta variación es que ocurrió en un término de 3 días, en que las fechas de cierre 11/12/17, 12/12/17 y 13/12/17 se alcanzaron precio de cierre de $\$ 3.030,00 ; \$ 3.364,00$ y $\$ 3.015,00$; respectivamente.

Pasando a los vencimientos mensuales, podemos identificar una mayor variación de $13,14 \%$ y una menor variación de $-10,65 \%$. Continuando con el vencimiento trimestral, identificamos variaciones de $+18,09 \%$ y $-25,13 \%$ en un trimestre y finalmente en los vencimientos semestrales, mostrando las mayores variaciones de los cuatro periodos analizados, encontramos exposiciones de $+15,37 \%$ y $-30,80 \%$.

Finalmente, podemos identificar que en los casos en que las empresas no gestionen estas exposiciones a riesgo, es muy probable que afecten su flujo de caja y, por ende, la liquidez, poniendo en peligro la perdurabilidad de la empresa.

\subsection{Análisis del precio forward USD/COP entre el 2011 y 2017}

Finalmente, analizaremos la eficiencia de los activos forward USD/COP de 0 a 1 mes, de 1 a 3 meses, forward USD/COP de 3 a 6 meses y activos de forward USD/COP mayores a 6 meses, basados en los datos reportados por la plataforma Set Fx de la Bolsa de Valores de Colombia y evaluados a través del modelo VaR.

Sobre 1.808 días que se cotizaron divisas USD/COP, en el mercado colombiano de divisas, el derivado con mayor disponibilidad fue el forward USD/COP de 3 a 6 que fue cotizado 1.365 días, seguida por el forward USD/COP mayor a 6 meses disponible 
Eficiencia del forward como instrumento de cobertura del riesgo cambiario en las empresas que realizan operaciones...

1.361 días y finalmente los activos forward USD/COP de 0 a 1 mes y de 1 a 3 meses, que estuvieron disponibles 1.344 y 1.340 días respectivamente, demostrando una baja disponibilidad como instrumento de cobertura, lo que condiciona su uso como instrumento de cobertura en riesgo de tasa de cambio.

Al analizar las correlaciones de las variables analizadas, el spot de fecha de apertura USD/COP, el spot USD/COP de fecha de cierre y los precios de los activos forward USD/ COP, encontramos una significancia de 0,01 y una correlación cercana a 1, lo que demuestra la eficiencia del instrumento como herramienta de cobertura, a pesar de su poca disponibilidad, ver tablas 3, 4, 5 y 6.

Tabla 3. Tabla de correlación forward 0 a 1 mes

\begin{tabular}{|c|c|c|c|c|}
\hline & & Spot & Forward 0 a 1 mes & Spot de cierre \\
\hline \multirow{3}{*}{ Spot } & Correlación de Pearson & 1 & $0,985^{* *}$ & $0,982^{* *}$ \\
\hline & Sig. (bilateral) & & 0,000 & 0,000 \\
\hline & N (Número de Observaciones) & 1.345 & 1.345 & 1.345 \\
\hline \multirow{3}{*}{$\begin{array}{c}\text { Forward } \\
0 \text { a } 1 \\
\text { mes }\end{array}$} & Correlación de Pearson & $0,985^{* *}$ & 1 & $0,968^{* *}$ \\
\hline & Sig. (bilateral) & 0,000 & & 0,000 \\
\hline & N (Número de Observaciones) & 1.345 & 1.345 & 1345 \\
\hline \multirow{3}{*}{$\begin{array}{l}\text { Spot de } \\
\text { cierre }\end{array}$} & Correlación de Pearson & $0,982^{* *}$ & $0,968^{* *}$ & 1 \\
\hline & Sig. (bilateral) & 0,000 & 0,000 & \\
\hline & N (Número de Observaciones) & 1.345 & 1.345 & 1.345 \\
\hline
\end{tabular}

** La correlación es significativa en el nivel 0,01 (bilateral).

Fuente: elaboración propia a partir de Set Fx.

Tabla 4. Tabla de correlación forward 1 a 3 meses

\begin{tabular}{|c|c|c|c|c|}
\hline & & Spot & Forward 1 a 3 meses & Spot de cierre \\
\hline \multirow{3}{*}{ Spot } & Correlación de Pearson & 1 & $0,995^{* *}$ & $1,000^{* *}$ \\
\hline & Sig. (bilateral) & & 0,000 & 0,000 \\
\hline & N (Número de Observaciones) & 1.340 & 1.340 & 1.340 \\
\hline \multirow{3}{*}{$\begin{array}{c}\text { Forward } \\
1 \text { a } 3 \\
\text { meses }\end{array}$} & Correlación de Pearson & $0,995^{* *}$ & 1 & $0,995^{* *}$ \\
\hline & Sig. (bilateral) & 0,000 & &, 000 \\
\hline & N (Número de Observaciones) & 1.340 & 1.340 & 1.340 \\
\hline \multirow{3}{*}{$\begin{array}{l}\text { Spot de } \\
\text { cierre }\end{array}$} & Correlación de Pearson & $1,000^{* *}$ & $0,995^{* *}$ & 1 \\
\hline & Sig. (bilateral) & 0,000 & 0,000 & \\
\hline & N (Número de Observaciones) & 1.340 & 1.340 & 1.340 \\
\hline
\end{tabular}

* La correlación es significativa en el nivel 0,01 (bilateral).

Fuente: elaboración propia a partir de Set Fx. 
Carlos Andrés Díaz Restrepo y Marlen Isabel Redondo Ramírez

Tabla 5. Tabla de correlación forward 3 a 6 meses

\begin{tabular}{|c|c|c|c|c|}
\hline & & Spot & $\begin{array}{c}\text { Forward } 3 \text { a } 6 \\
\text { meses }\end{array}$ & Spot de cierre \\
\hline \multirow{3}{*}{ Spot } & Correlación de Pearson & 1 & $0,995^{* *}$ & $1,000^{* *}$ \\
\hline & Sig. (bilateral) & & 0,000 & 0,000 \\
\hline & N (Número de Observaciones) & 1.365 & 1.365 & 1.365 \\
\hline \multirow{3}{*}{$\begin{array}{c}\text { Forward } \\
3 \text { a } 6 \\
\text { meses }\end{array}$} & Correlación de Pearson & $0,995^{* *}$ & 1 & $0,995^{* *}$ \\
\hline & Sig. (bilateral) & 0,000 & & 0,000 \\
\hline & N (Número de Observaciones) & 1.365 & 1.365 & 1.365 \\
\hline \multirow{3}{*}{$\begin{array}{l}\text { Spot de } \\
\text { cierre }\end{array}$} & Correlación de Pearson & $1,000^{* *}$ & $0,995^{* *}$ & 1 \\
\hline & Sig. (bilateral) & 0,000 & 0,000 & \\
\hline & N (Número de Observaciones) & 1.365 & 1.365 & 1.365 \\
\hline
\end{tabular}

•*. La correlación es significativa en el nivel 0,01 (bilateral).

Fuente: elaboración propia a partir de Set Fx.

Tabla 6. Tabla de correlación forward mayor a 6 meses

\begin{tabular}{|c|c|c|c|c|}
\hline & & Spot & $\begin{array}{c}\text { Forward mayor } \\
\text { a } 6 \text { meses }\end{array}$ & Spot de cierre \\
\hline \multirow[t]{3}{*}{ Spot } & Correlación de Pearson & 1 & $0,991^{* *}$ & $1,000^{* *}$ \\
\hline & Sig. (bilateral) & & 0,000 & 0,000 \\
\hline & N (Número de Observaciones) & 1.361 & 1.361 & 1.361 \\
\hline \multirow{3}{*}{$\begin{array}{l}\text { Forward } \\
\text { mayor a } \\
6 \text { meses }\end{array}$} & Correlación de Pearson & $0,991^{* *}$ & 1 & $0,991^{* *}$ \\
\hline & Sig. (bilateral) & 0,000 & & 0,000 \\
\hline & N (Número de Observaciones) & 1.361 & 1.361 & 1.361 \\
\hline \multirow{3}{*}{$\begin{array}{l}\text { Spot de } \\
\text { cierre }\end{array}$} & Correlación de Pearson & $1,000^{* *}$ & $0,991^{* *}$ & 1 \\
\hline & Sig. (bilateral) & 0,000 & 0,000 & \\
\hline & N (Número de Observaciones) & 1.361 & 1.361 & 1.361 \\
\hline
\end{tabular}

•*. La correlación es significativa en el nivel 0,01 (bilateral).

Fuente: elaboración propia a partir de $\operatorname{Set} F x$.

Al comparar los precios de cierre de los activos forward de 0 a 1 mes, de 1 a 3 meses, de 3 a 6 meses y mayor a 6 meses, podemos verificar que el comportamiento en los diferentes periodos es diferente, a pesar de que tienen una buena correlación, presentan una baja eficiencia como instrumento de cobertura, debido a sus grandes rangos de diferencia con los precios spot de cierre. 
Eficiencia del forward como instrumento de cobertura del riesgo cambiario en las empresas que realizan operaciones...

\section{Gráfica 3. Precios de cierre forward del 01-01-2011 al 31-12-2017}

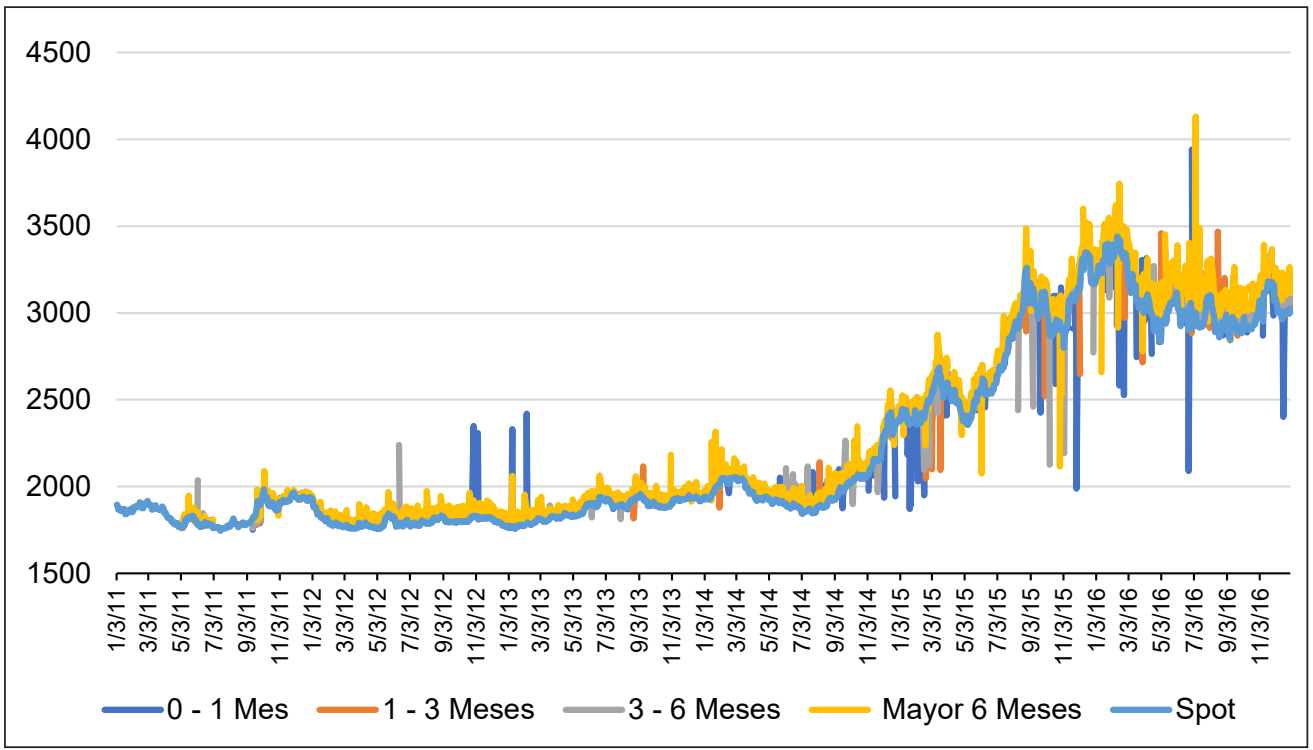

\section{Fuente: elaboración propia a partir de Set Fx.}

El comportamiento del precio de los forward se resume en la tabla 7, en esta podemos observar que en el 53,53 \% de los casos revisados el precio spot de cierre del USD/COP, estuvo por encima del precio de cierre del forward de 0 a 1 mes, seguido del 12,61 \% el forward de 1 a 3 meses tuvo el mismo comportamiento, y finalmente en el 7,03 \% de los casos en forward de 3 a 6 meses y el 5,66\% en los eventos revisados en el forward mayor a 6 meses, los precios de cierre de la divisa USD al contado fueron mayores que los contratos de derivados forward USD/COP.

\section{Tabla 7. Tabla resumen precios de forward}

\begin{tabular}{lrrrrr}
\hline Descripción & Spot & $\begin{array}{c}\text { Forward 0 a } 1 \\
\text { mes }\end{array}$ & $\begin{array}{c}\text { Forward 1 a } 3 \\
\text { meses }\end{array}$ & $\begin{array}{c}\text { Forward 3 a 6 } \\
\text { meses }\end{array}$ & $\begin{array}{c}\text { Forward mayor } \\
\text { a 6 meses }\end{array}$ \\
\hline Máximo & $\$ 3.441,00$ & $\$ 3.932,00$ & $\$ 3.479,77$ & $\$ 3.548,51$ & $\$ 4.131,07$ \\
\hline Mínimo & $\$ 1.746,00$ & $\$ 1.750,95$ & $\$ 1.755,80$ & $\$ 1.761,62$ & $\$ 1.760,00$ \\
\hline Desviación & $\$ 533,02$ & $\$ 509,10$ & $\$ 515,44$ & $\$ 532,79$ & $\$ 554,74$ \\
\hline $\begin{array}{l}\text { Spot cierre }> \\
\text { forward }\end{array}$ & & $53,53 \%$ & $12,61 \%$ & $7,03 \%$ & $5,66 \%$ \\
\hline
\end{tabular}

\section{Fuente: elaboración propia a partir de Set Fx.}

En la tabla 7, resumiendo los precios de cierre de los forwards, podemos identificar que no existe ninguna similitud en ninguno de los activos, generando precios alta- 
mente costosos comparados con el mayor precio de spot de cierre, lo que aumenta el costo de transacción en las operaciones de cobertura de riesgo con este activo.

Finalmente, comparando el precio spot de cierre al final del forward, podemos encontrar un porcentaje muy bajo en los que el forward fue menor que el spot, evidenciando que este activo de cobertura es óptimo para compras (importaciones) en dólares más no para ventas (exportaciones) en dólares.

\section{CONCLUSIONES}

Las empresas que realizaron operaciones en dólares estuvieron expuestas a un alto riesgo en tasa de cambio durante los años 2011 a 2017. A medida que es más larga la exposición al riesgo, mayor es la diferencia, pasando por una exposición diaria de $+1,93 \%$, mensual de $+8,94 \%$, trimestral de $+13,31 \%$ y semestral de $+17,7 \%$.

Esto se resume en un mayor o menor costo en las importaciones y un mayor o menor ingreso en las exportaciones, para las empresas que hacen operaciones en dólares.

La mayor exposición positiva fue del $+18,08 \%$ en el trimestre comprendido entre el 02 de noviembre de 2015 y cierre el 02 de febrero de 2017 y la mayor exposición negativa fue del $-30,80 \%$ en el semestre del 17 de marzo de 2014 al cierre 17 de septiembre de 2017.

Al evaluar el comportamiento de los instrumentos de cobertura forward, durante los periodos 2011 al 2017, podemos evidenciar que existe una baja liquidez en estos activos, condicionando la disponibilidad de instrumentos de cobertura para la gestión del riesgo en tasa de cambio USD/COP.

El activo con mayor disponibilidad es el forward, sin embargo, es el de menor correlación frente a los precios spot de cierre, lo que evidencia un comportamiento diferente entre los precios de este activo y el dólar al contado. Este activo puede ser más eficiente para cobertura en procesos de exportación dado que en al menos el $12 \%$ de los eventos revisados, el precio de cierre del forward es mayor al spot de cierre al vencimiento del contrato.

Posterior a esto, el Banco de la República debe crear generadores de mercado, similares a los generados de compra y venta de divisas al contado, con el fin de garantizar una mayor liquidez en los forwards. Igualmente, debe facilitar que los intermediarios del mercado cambiarios, como lo son las entidades financieras o los comisionistas de bolsa, faciliten la disponibilidad de activos con bajo precio, sin condicionar valores mínimos de contratos tan altos, debido a que restringe el acceso a las pymes. 
Eficiencia del forward como instrumento de cobertura del riesgo cambiario en las empresas que realizan operaciones...

\section{BIBLIOGRAFÍA}

Agudelo Rueda, Diego. (2014). Inversiones en renta variable: fundamentos y aplicaciones al mercado accionario colombiano. Medellín: Fondo Editorial Universidad Eafit, 474 p.

Banco de la República. (2013). ¿¿ué es la tasa de cambio? Bogotá: Banrepública.

Bolsa de Valores de Colombia. (2008). Guía colombiana del mercado de valores. Bogotá: Colombia, 250p.

BVC. (2017). Integración Corporativa Deceval - BVC. Bogotá: BBV, 12p.

Carbaugh, R. (2016). Economía internacional. México: Gengage Learning. 250 p.

Comisión Nacional del Mercado de Valores. (2015). Los Fondos Cotizados (ETF). Bogotá: BBVA.

DIAN -Dirección de Impuestos y Aduanas Nacionales-. (2019). Preguntas frecuentes sobre el control cambiario. Bogotá: DIAN.

Fradique-Méndez, Carlos. (2014). Guía del mercado de valores. Bolsa de Valores de Colombia. $113 \mathrm{p}$.

García, Pablo y Díez, Luis. (2014). Mercados financieros internacionales. Madrid: Delta Publicaciones. 251 p.

Grath, Anders. (2016). The Handbook of International Trade and Finance. Londres: Kogan Page, $288 \mathrm{p}$.

De Jesús, Raúl; Ortiz, Edgar y Cabello, Alejandro. (2013). Long run peso/dollar exchange rates and extreme value behavior: Value at Risk modeling. En: The North American Journal of Economics and Finance, vol. 24, p. 139-152. DOI: 10.1016/j.najef.2012.06.001.

Jorion, Philippe. (2000). Valor en riesgo. Ciudad de México: Limusa. 357 p.

Jorion, Philippe. (1990). The Exchange-Rate Exposure of U. S. Multinationals. En: The Journal of Business, vol. 63, p. 331-345.

Kozikowski, Zbigniew. (2013). Finanzas internacionales. Toluca: McGraw-Hill, 300 p.

Krugman, Paul y Obstfeld, Maurice. (2012). Economía internacional. Teoría y política. Madrid: Pearson. 334 p.

Jacque, Laurent (2014). International Corporate Finance. New Jersey: Wiley, 360p.

McLeay, Michael; Radia, Amar y Thomas, Ryland. (2015). La creación del dinero en la economía moderna. En: Revista de Economía Institucional, vol. 17, n. 33, p. 355-383.

Superintendencia Financiera de Colombia. (2017). Conformación del sistema financiero colombiano. Bogotá: SFC, 2 p.

Toro Díaz, Jairo; Redondo Ramírez, Isabel y Díaz Restrepo, Carlos. (2015). Riesgo Financiero en las empresas de la ciudad de Medellín durante el año 2013. En: Revista Gestión y Región, vol. 20, p. 139-159.

Uribe, José Darío. (2006). El mercado monetario en Colombia. En: Revista del Banco de la República, vol. 79 , n. 944, p. 1 - 9. 
Uribe, José Darío. (2013). El sistema financiero colombiano: estructura y evolución reciente. En: Revista del Banco de la República, vol. 86, n. 1023, p. 5-15.

Wang, Zongrun; Wu, Weitao; Chen, Chao y Zhou, Yanju. (2010). The exchange rate risk of Chinese yuan: Using VaR and ES based on extreme value theory. En: Journal of Applied Statistics, vol. 37, n. ${ }^{\circ}$ 2, p. 265-282. DOI: 10.1080/02664760902846114

Ye, Min; Hutson, Elaine y Muckley, Cal. (2014). Exchange rate regimes and foreign exchange exposure: The case of emerging market firms. En: Emerging Markets Review, vol. 21, p. 156-182. doi.org/10.1016/j.ememar.2014.09.001

Zhou, Li; Zhang, Ning y Chen, Qing-yi. (2013). Value-at-Risk Modelling for Risk Management of RMB Exchange Rate. En: International Journal of Applied Mathematics and Statistics, vol. 43, n. ${ }^{\circ} 13$, p. 297-304. 